\title{
Assessing the utility of virtual OSCE sessions as an educational tool: a national pilot study
}

\author{
Sarika Grover \\ King's College London \\ Maharsh Pandya \\ Cardiff University \\ Chavini Ranasinghe \\ University College London

\section{Saajan P. Ramji} \\ King's College London \\ Harroop Bola ( $\sim$ Harroop.bola19@Imperial.ac.uk) \\ Imperial College School of Medicine, Imperial College London \\ Siddarth Raj \\ King's College London
}

\section{Research Article}

Keywords: Virtual, Objective structured clinical examination (OSCE), Educational tool, National pilot study

Posted Date: August 24th, 2021

DOl: https://doi.org/10.21203/rs.3.rs-800792/v1

License: (c) (i) This work is licensed under a Creative Commons Attribution 4.0 International License. Read Full License

Version of Record: A version of this preprint was published at BMC Medical Education on March 15th, 2022. See the published version at https://doi.org/10.1186/s12909-022-03248-3. 


\section{Abstract}

\section{Background}

Objective Structured Clinical Examinations (OSCEs) are a common form of assessment used across medical schools in the UK to assess clinical competence and practical skills and are traditionally held in an in-person format. In the past, medical students have often prepared for such exams through in-person peer-assisted learning (PAL), however, due to the recent Covid-19 pandemic, many in-person teaching sessions transitioned to online-based formats. There is currently a paucity of research on the utility of virtual PAL OSCE sessions and thus, we carried out a national pilot study to determine the effectiveness of virtual OSCE teaching via feedback from participants and examiners.

\section{Methods}

A total of 85 students attended from 19 UK-based medical schools with eight students based internationally attended the series of online OSCE workshops delivered via Zoom ${ }^{\square}$. All students and examiners completed a feedback questionnaire at the end of each session regarding parameters, which included questions on pre-and post-workshop confidence in three OSCE domains: history-taking, communication and data interpretation. The five-point Likert scale was used to self-report confidence, and the results were analysed using the Mann-Whitney $U$ test after assessing for normality using the Shapiro-Wilk test.

\section{Results}

Results from student feedback showed an increase in confidence for all three OSCE domains after each event $(p<0.001$ ) with $69.4 \%$ agreeing or strongly agreeing that online OSCE sessions could sufficiently prepare them for in-person exams. Questionnaire feedback revealed that $97.6 \%$ of students and $86.7 \%$ of examiners agreed that virtual OSCE teaching would be useful for preparing for in-person OSCE examinations after the pandemic.

\section{Conclusion}

The majority of participants in the virtual OSCE sessions reported an improvement in their confidence in history-taking, communication and data interpretation skills. The majority of participants and examiners also reported that they found virtual OSCE sessions to be as engaging and as interactive as in-person teaching. This study has demonstrated that virtual OSCE workshops can serve as a valuable learning resource for students with the potential to be beneficial beyond the pandemic, however, further studies that directly compare academic outcomes between in-person and virtual OSCE teaching sessions are required.

\section{Introduction}


Objective structured clinical examinations (OSCEs), since being introduced in 1975, have become a widely recognised form of assessment across medical schools in the United Kingdom (UK) [1]. This inperson examination requires students to rotate between a series of stations, each of which usually lasts between six to 15 minutes [2,3]. Each station typically assesses a core skill or combination of skills that include, but are not limited to, simulated consultations, clinical examinations, practical procedures and clinical data interpretation $[2,3]$.

Clinical teaching in the format of peer-assisted learning (PAL) is a common form of OSCE preparation [4]. Two separate studies assessing the effectiveness of in-person PAL OSCE teaching by Rashid et al. and Bevan et al. concluded that in-person PAL OSCE teaching was not only useful for all participants but significantly improved participant confidence with students stating that they felt 'better equipped' for their OSCEs $[5,6]$.

The recent Coronavirus (COVID-19) pandemic has severely restricted the ability to organise and deliver inperson teaching, and many aspects of medical education have been forced to shift to online learning via platforms such as Zoom as a way to reduce social contact $[7,8]$. Given the importance placed on OSCEs by medical schools, along with the fact that in-person OSCE teaching was not possible due to the ongoing pandemic, virtual sessions have served as a potentially viable alternative for delivering OSCE teaching.

One study by Prettyman et al. that involved the delivery of virtual OSCE teaching for nurse practitioner students in America stated that some key advantages in comparison to in-person teaching included: ease of attendance despite physical distance, practicing and teaching relevant skills for virtual consultations for future virtual clinics and the ease of setup in comparison to in-person teaching [9].

Currently, there is a scarcity of research on the effectiveness of virtual OSCE teaching for medical students. Existing studies in the UK mainly focus on virtual OSCE teaching specific to a given medical school [4]. Therefore, while there is emerging literature on the trial and delivery of virtual OSCE teaching, there is still a paucity of evidence regarding the advantages and disadvantages in comparison to inperson OSCE teaching, specific to all medical students nationwide $[10,11]$.

During the height of the COVID-19 pandemic, a few medical schools opted to deliver final year OSCEs in a virtual format, while some chose to postpone or cancel them altogether [12]. However, with the UK easing COVID-19 restrictions, it is likely that in-person OSCE examinations are bound to resume nationwide, and hence we must reconsider whether virtual OSCE teaching has a role to play once the necessity for virtual teaching and examining diminishes.

Therefore, this pilot study aims to use participant feedback to assess the impact of a nationally delivered virtual OSCE teaching series on participant confidence, the comparison between previous in-person teaching and the scope for future virtual OSCE teaching in a post-pandemic landscape.

\section{Methods}


The methods in this study were performed following the STROBE guidelines for reporting observational studies, which include a checklist for cross-sectional studies [13].

\section{Workshops}

The national OSCE teaching was delivered as a series of online workshops delivered on a weekly basis via Zoom ${ }^{\square}$ (Zoom Video Communications, USA), from February to March 2021 as a nationally organised student-led initiative. It was identified that there was a deficit in the availability of online structured OSCE teaching that supported the student experience with both practice and detailed feedback. Therefore, launching an OSCE workshop series was an appropriate medium to widen accessibility and utility to improve clinical practice. The sessions focused on practising common OSCE stations, including historytaking, communication and data interpretation skills. Sessions were delivered for each of the commonly examined specialities: cardiology, respiratory, gastroenterology, neurology and endocrinology.

In total, the series was composed of five consecutive workshops, attended by 85 students distributed throughout. Each session had a total of six OSCE stations and consisted of two circuits with three stations per circuit. A single circuit composed of one history-taking station, one communication station and one data interpretation station, each of which was overseen by an assigned examiner. The examiners were clinical-year medical students at various medical schools across the UK and they led, facilitated and delivered feedback for their assigned station. The maximum number of students in each session was limited to 36 in order to ensure that the examiners were able to provide detailed and individualised feedback within the allocated time. The students signed up for the workshops via an online application form and were allocated to each session on a first come, first served basis.

The format remained the same for both the history-taking and communication stations. Resources were prepared in advance by the examiners and students were assigned pairs within their groups of six; each pair then took it in turn to practice a scenario. One student would take on the role of the patient while the other would serve as the active participant. The role of the student would alternate between the communication and history-taking stations, thereby giving students the opportunity to both practice skills and contribute to the feedback for their peers. Simultaneously, the examiners would review participant performance during the stations and provide individualised feedback, thereby opening space for reflection. At the end of each session, the examiners would collectively go over the predesigned mark scheme with each group of six with the aim of exploring how to approach a similar station. The data interpretation station was delivered in a group-teaching format, whereby the examiners discussed scenarios with the students and offered methods, advice and tips on tackling a similar station in the exam. 
At the end of each workshop, each of the students was given a feedback questionnaire which asked them, in the form of comparative statements, to state how strongly they agreed with the following: online OSCE teaching is as interactive, as engaging, as useful in enabling to them develop relevant clinical skills and providing constructive and helpful feedback. The questionnaire also asked the students to rank their confidence in history-taking, communication and data interpretation for that specialty before and after the session, which was graded on a 5-point Likert scale where $1=$ not confident at all and $5=$ very confident. A Likert scale was chosen to quantify students' perceived confidence. Importantly, students were also asked whether they that online OSCE sessions would be useful in the post-pandemic phase.

\section{Examiner feedback}

The examiners were given similar questionnaires asking them to state how strongly they agreed or disagreed with the same comparative statements regarding online and in-person OSCE teaching, and whether they felt that online-based OSCE teaching would be useful for learning after the pandemic.

\section{Statistical methods}

Attendees completed post-workshop questionnaires and data was collected regarding the demographics of the students and feedback of the workshops. Five-point Likert scales were used to quantify the qualitative opinions and perceptions of students and examiners. The Shapiro-Wilk test was used to assess whether the data was normally distributed, and the Mann-Whitney $U$ test was used to assess statistical differences between nonparametric pre- and post-event responses.

\section{Ethics}

This study involved no patients and consisted entirely of both preclinical and clinical medical students attending an undergraduate student-led workshop, requiring no medical or personal information. All participants in this study gave approval for their anonymous data to be used towards potential future research.

\section{Results}

\section{Students}

A total of 85 students attended the five sessions with an average of 17 (10-22) students per session, of these students, 64 identified as female and 21 as male. These students included 57 (67.1\%) clinical 
medical students and 28 (32.9\%) pre-clinical medical students across 19 UK-based medical schools, with eight students attending from medical schools outside of the UK. This is demonstrated in Table 1.

Table 1

- Baseline characteristics of participants attending the five virtual OSCE sessions

\begin{tabular}{|ll|}
\hline Parameter & $\mathbf{N}=\mathbf{8 5}$ \\
\hline Sex & $64(75.3 \%)$ \\
Male & $21(24.7 \%)$ \\
Prefer not to say & $0(0 \%)$ \\
Stage of Training & \\
Pre-clinical & $28(32.9 \%)$ \\
Clinical & $56(65.9 \%)$ \\
Intercalating & $1(0.01 \%)$ \\
Location of medical school & \\
UK-based & $77(90.6 \%)$ \\
International & $8(0.10 \%)$ \\
\hline Received previous in-person OSCE teaching? & $59(69.4 \%)$ \\
Yes & $26(30.6 \%)$ \\
No & \\
\hline
\end{tabular}

Students were asked if they had previously received in-person OSCE teaching at any point prior to the virtual event, to which $59(69.4 \%)$ of students stated that they had. Of those who had received in-person OSCE teaching, 48 (81.4\%) agreed or strongly agreed that virtual OSCE teaching was as engaging, 49 $(83.1 \%)$ agreed or strongly agreed that it was as interactive and $48(81.4 \%)$ agreed or strongly agreed that it enabled them to further develop and enhance their previous history-taking, communication and data interpretation skills. Finally, $52(88.1 \%)$ students agreed or strongly agreed that OSCE teaching provided them with appropriate feedback on their performance for preparation of medical school OSCEs.

Most students (69.4\%) agreed or strongly agreed that online OSCE sessions could sufficiently prepare them for in-person OSCE examinations, and 83 (97.6\%) students agreed that virtual OSCE teaching after the pandemic would be useful for preparation for medical school examinations.

The effect of the virtual OSCE teaching sessions on participants' confidence has been reported with preand post-event medians along with statistical significance and effect sizes (Table 2). This indicated that 
there was a statistically significant improvement in participants' confidence across all three OSCE domains as a result of attending the virtual OSCE sessions $(p<0.0001)$.

Table 2

- Effect of virtual OSCE teaching sessions on participants confidence with statistical significance and effect size

\begin{tabular}{|c|c|c|c|c|}
\hline OSCE Domain & $\begin{array}{l}\text { Pre-session confidence } \\
\text { (median) }\end{array}$ & $\begin{array}{l}\text { Post-session confidence } \\
\text { (median) }\end{array}$ & $\begin{array}{l}\mathrm{p}- \\
\text { value }\end{array}$ & $\begin{array}{l}\text { Effect } \\
\text { size (r) }\end{array}$ \\
\hline History Taking & 3 & 4 & $<.0001$ & 1.373748 \\
\hline Communication & 3 & 4 & $<.0001$ & 1.662572 \\
\hline Data Analysis & 3 & 4 & $<.0001$ & 1.747822 \\
\hline
\end{tabular}

\section{Examiners}

A total of 15 different examiners assisted in the virtual OSCE workshops with six examiners assisting in each workshop. The examiners consisted of 11 female and four male medical students in their clinical years across seven different UK-based medical schools. All examiners had previously undertaken an average of two formative OSCES and one summative OSCE at their respective medical schools. Seven (46.7\%) examiners had previous experience in delivering face-to-face OSCE teaching prior and all examiners had previously received either face-to-face OSCE teaching during medical school or smallscale virtual OSCE teaching prior to this workshop.

In comparison to the students that attended, 11 (73.3\%) examiners agreed or strongly agreed that virtual OSCE teaching was as engaging as in-person OSCE teaching, and eight (53.3\%) examiners agreed or strongly agreed that it was as interactive. Thirteen (86.7\%) examiners agreed that virtual OSCE teaching after the pandemic would be useful for preparation for medical school examinations.

\section{Discussion}

There has been a clear shift to online teaching as a result of the global COVID-19 pandemic, and virtual OSCE assessments organised by medical schools have been found to retain the same ability to assess communication skills, history-taking, clinical reasoning and formulating differential diagnoses and management plans as traditional face-to-face teaching [9]. Additional advantages over traditional inperson group teaching includes the reduced travelling time, which could allow students to spend more time learning, whilst providing similar benefits to in-person teaching but in a more comfortable environment [14]. Virtual OSCE sessions also have the benefit of running larger and more regular sessions without physical constraints, such as booking rooms. 
The overwhelmingly positive feedback received from students and the examiners indicated that this model of virtual mock OSCE delivery was valuable and feasible for both groups. As per the feedback, 81$83 \%$ of students agreed or strongly agreed that the sessions were interactive and engaging, which is particularly important when considering the feasibility of shifting to an online format. Additionally, $97.6 \%$ agreed that online teaching would be valuable for exams, and this is further supported by the statistically significant improvement in confidence after these virtual sessions across all three domains: historytaking communication and data-analysis (Table 2).

Moreover, studies running similar virtual OSCEs have shown that it is cost-efficient, useful and feasible in the current global situation with a potential role even in the post-pandemic phase [15]. As our OSCE workshops were all online, we were able to reach students on an international level, thus providing students from across the world with the opportunity to participate and access resources that they may have otherwise been unable to reach if the sessions were run in-person.

Another potential benefit of this format was the peer-led nature of the sessions, which could have helped examiners consolidate their own knowledge in a format that was perceived to be interactive, engaging and beneficial for OSCE preparation. This advantage has been demonstrated previously, where near-peer teaching has been shown to provide a mutually beneficial solution to all students involved, by consolidating knowledge for both tutors and tutees, whilst fostering confidence as educators [16].

Additionally, a significant drawback of the virtual format was that students were unable to practise examination skills and practical procedures online, both of which are essential skills required for summative OSCEs and clinical practice. It is possible that future studies could try to assess the feasibility of adopting a hybrid format or utilising virtual sessions to practice examinations and procedures. Moreover, further studies that directly compare academic outcomes between in-person and virtual OSCE teaching sessions are required to understand if there are any significant differences between the two teaching formats. Therefore, at present, it is not possible to comment on whether online teaching can fully replace clinical face-to-face teaching $[14,17]$.

Furthermore, although all five sessions were deemed to be successful, there were anecdotal issues to note, particularly with timing when hosting sessions for larger groups of students. This was due mainly to internet connection issues, which led to audio lag and difficulty accessing online resources during both the station and feedback sessions. Similar issues have been highlighted within the literature [10], with a systematic review of medical education during the COVID-19 pandemic showing that virtual teaching poses unique challenges, including reduced student engagement compared to in-person sessions [18]. We anticipate that replicating our workshops on a larger scale would amplify these issues and would require more time, preparation and administrative power to overcome. Other studies have further emphasised how planning and preparation is key to the smooth running of virtual OSCEs [10].

Moving forwards, overcoming these issues could be achieved by incorporating guidance from published literature on how to run virtual OSCEs by building on the lessons learnt from other organisations that have created similar online teaching programmes $[11,19]$. 


\section{Conclusion}

In summary, this national pilot study has demonstrated that virtual OSCE workshops can serve as a valuable learning resource for students, with the potential to be beneficial beyond the pandemic. The peer-led virtual OSCE sessions were deemed by the majority of students and examiners alike to be as interactive and engaging, with the majority of students reporting an improvement in confidence in historytaking, communication and data interpretation skills. The majority of students and examiners and also agreed that they would find virtual OSCE sessions to be useful when preparing for summative OSCES even after the pandemic.

\section{References}

1. Harden RM, Stevenson M, Downie WW, Wilson GM. Assessment of clinical competence using objective structured examination. Br Med J. 1975;1(5955):447-451. doi:10.1136/bmj.1.5955.447

2. Queen's University Belfast. OSCE Training - What are OSCEs? 2021. Accessed 4 June 2021. Available from: https://www.med.qub.ac.uk/osce/background_What.html

3. University of Liverpool. Introduction to OSCEs. 2021. Accessed 4 June 2021. Available from: https://www.liverpool.ac.uk/medicine/osce/introductiontoosces/

4. Khan R, Payne MWC, Chahine S. Peer assessment in the objective structured clinical 21 examination: A scoping review. Med Teach. 2017;39(7):745-756. doi:10.1080/0142159X.2017.1309375

5. Rashid MS, Sobowale O, Gore D. A near-peer teaching program designed, developed and delivered exclusively by recent medical graduates for final year medical students sitting the final objective structured clinical examination (OSCE). BMC Med Educ. 2011;11:11. doi:10.1186/1472-6920-11-11

6. Bevan J, Russell B, Marshall B. A new approach to OSCE preparation - PrOSCEs. BMC Med Educ. 2019;19(1):126. doi:10.1186/s12909-019-1571-5

7. Mian A, Khan S. Medical education during pandemics: a UK perspective. BMC Med. 2020;18(1):100. doi:10.1186/s12916-020-01577-y

8. Daniel $\mathrm{M}$, Gordon $\mathrm{M}$, Patricio $\mathrm{M}$, et al. An update on developments in medical education in response to the COVID-19 pandemic: A BEME scoping review: BEME Guide No. 64. Med Teach. 2021;43(3):253-271. doi:10.1080/0142159X.2020.1864310

9. Prettyman AV, Knight EP, Allison TE. Objective Structured Clinical Examination From Virtually Anywhere! Journal for Nurse Practitioners. 2018;14(8):e157-e163. https://doi.org/10.1016/j.nurpra.2018.05.007

10. Hannan TA, Umar SY, Rob Z, Choudhury RR. Designing and running an online Objective Structured Clinical Examination (OSCE) on Zoom: A peer-led example. Med Teach. 2021;43(6):651-655. doi:10.1080/0142159X.2021.1887836

11. Hopwood J, Myers G, Sturrock A. Twelve tips for conducting a virtual OSCE. Med Teach. 2021;43(6):633-636. doi:10.1080/0142159X.2020.1830961 
12. Blythe, J., Patel, N.S.A., Spiring, W. et al. Undertaking a high stakes virtual OSCE ("VOSCE") during Covid-19. BMC Med Educ 21, 221 (2021). Doi:10.1186/s12909-021-02660-5

13. von Elm E, Altman DG, Egger M, et al. The Strengthening the Reporting of Observational Studies in Epidemiology (STROBE) statement: guidelines for reporting observational studies. J Clin Epidemiol. 2008;61(4):344-349. doi:10.1016/j.jclinepi.2007.11.008

14. Dost S, Hossain A, Shehab M, Abdelwahed A, Al-Nusair L. Perceptions of medical students towards online teaching during the COVID-19 pandemic: a national cross-sectional survey of $2721 \mathrm{UK}$ medical students. BMJ Open. 2020;10(11):e042378. doi:10.1136/bmjopen-2020-042378

15. Arrogante O, López-Torre EM, Carrión-García L, Polo A, Jiménez-Rodríguez D. High-Fidelity Virtual Objective Structured Clinical Examinations with Standardized Patients in Nursing Students: An Innovative Proposal during the COVID-19 Pandemic. Healthcare (Basel). 2021;9(3):355. doi:10.3390/healthcare9030355

16. Cash T, Brand E, Wong E, Richardson J, Athorn S, Chowdhury F. Near-peer medical student simulation training. Clin Teach. 2017;14(3):175-179. doi:10.1111/tct.12558

17. Hameed T, Husain M, Jain SK, Singh CB, Khan S. Online Medical Teaching in COVID-19 Era: Experience and Perception of Undergraduate Students. Maedica (Bucur). 2020;15(4):440-444. doi:10.26574/maedica.2020.15.4.440

18. Wilcha RJ. Effectiveness of Virtual Medical Teaching During the COVID-19 Crisis: Systematic Review. JMIR Med Educ. 2020;6(2):e20963. doi:10.2196/20963

19. Shehata MH, Kumar AP, Arekat MR, et al. A toolbox for conducting an online OSCE. Clin Teach. 2021;18(3):236-242. doi:10.1111/tct.13285

\section{Declarations}

\section{Ethics approval and consent to participate}

Ethical approval was not required for this study as deemed by the University College London (UCL) Ethics committee. All participants gave their consent for their feedback to be involved in this study. The methods in this study were performed following the STROBE guidelines for reporting observational studies, which include a checklist for cross-sectional studies.

\section{Consent for publication}

Not applicable.

\section{Availability of data and material}


The data that support the findings of this study are available on request from the corresponding author, Harroop Bola.

\section{Competing interests}

All authors certify that they have no affiliations with or involvement in any organization or entity with any financial interest or non-financial interest in the subject matter or materials discussed in this manuscript.

\section{Funding}

The authors did not receive any support or funding from any organization for the submitted work.

\section{Authors' contribution}

SG, MP, and CR conceptualised the idea, organised and created the online workshop events. The resources used for teaching in each workshop were created by SG, MP, CR and SPR. SG and MP created the questionnaire for both the students and examiners. SG, MP, and SR reviewed the data and performed statistical analysis. SG, MP, CR, SPR and HB coordinated and equally contributed to writing the manuscript. SR oversaw, reviewed, and edited the manuscript. All authors read and approved the final manuscript for submission.

Conceptualization: SG, MP, and CR. Methodology: SG, MP, and CR. Investigation: SG, MP and CR. Visualization: SG, MP and CR. Formal analysis: SG, MP and CR. Writing - Original Draft: SG, MP, CR, SPR and HB. Writing - Review and Editing: SG and SR. Supervision: SR. Project Administration: SR.

\section{Acknowledgments}

Not applicable.

\section{Supplementary Files}

This is a list of supplementary files associated with this preprint. Click to download.

- AppendixAOSCE.docx

- AppendixBOSCE.docx 